\title{
Daidzein exerts anticancer activity towards SKOV3 human ovarian cancer cells by inducing apoptosis and cell cycle arrest, and inhibiting the Raf/MEK/ERK cascade
}

\author{
FU HUA $^{1}$, CHANG-HUA LI ${ }^{1}$, XIAO-GANG CHEN ${ }^{2}$ and XIAO-PING LIU ${ }^{1}$ \\ Departments of ${ }^{1}$ Gynecology and ${ }^{2}$ Orthopaedics, Huai'an First People's Hospital, Nanjing Medical University, \\ Huai'an, Jiangsu 223300, P.R. China
}

Received August 22, 2017; Accepted January 5, 2018

DOI: $10.3892 /$ ijmm.2018.3531

\begin{abstract}
Ovarian cancer is the main cause of gynecological cancer-associated mortality around the world. Despite initial responses to chemotherapy, frequent relapse occurs. Daidzein is an important flavonoid and has been shown to exhibit a diversity of pharmacological properties, including antimicrobial and anticancer activities. However, information on the anticancer activity of daidzein against ovarian cancer remains limited. Therefore, the present study evaluated the anticancer activity of daidzein against a panel of human ovarian cancer cell lines and one normal ovarian cell line (Moody). The results revealed that daidzein exhibited potent anticancer activity against SKVO3 cells with a half-maximal inhibitory concentration $\left(\mathrm{IC}_{50}\right)$ of $20 \mu \mathrm{M}$. However, it exhibited comparatively lower activity against normal ovarian Moody cells, which had an $\mathrm{IC}_{50}$ of $100 \mu \mathrm{M}$. Daidzein induced morphological changes in SKOV3 cells and mitochondrial apoptosis, as evident from DAPI, AO/EB and Annexin V/propidium iodide staining. This was associated with the upregulation of B-cell lymphoma 2-associated X protein, cytochrome $c$, cleaved caspase-3 and -9 , and cleaved poly (ADP-ribose) polymerase. Daidzein also triggered $\mathrm{G} 2 / \mathrm{M}$ cell arrest through the downregulation of pCdc25c, Cdc25c, pCdc2, Cdc2 and cyclin B1. The effect of daidzein on the migration of SKOV3 cells was also determined, the results of which indicated that daidzein inhibited cell migration in a concentration-dependent manner and was coupled with concomitant decrease in the expression of matrix metalloproteinase (MMP)-2 and -9 . Additionally, daidzein-inhibited cell growth was simultaneous with suppression of the expression of phosphorylated mitogen-activated protein kinase kinase and phosphorylated
\end{abstract}

Correspondence to: Dr Fu Hua, Department of Gynecology, Huai'an First People's Hospital, Nanjing Medical University, 6 Beijing Road West, Huai'an, Jiangsu 223300, P.R. China

E-mail: fidelazahmeliol@yahoo.com

Key words: ovarian cancer, daidzein, Raf/mitogen-activated protein kinase kinase/extracellular signal-regulated kinase, apoptosis extracellular signal-regulated kinase. The present study also examined whether daidzein exerts similar activity against SKOV3 cells in nude mouse xenograft models and it was revealed that daidzein considerably reduced the tumorigenesis in vivo, indicative of the potential for daidzein as a lead molecule in the development of ovarian cancer chemotherapy

\section{Introduction}

Ovarian cancer is one of the most life-threatening gynecological malignancies. It accounts for $\sim 3 \%$ of all cancer cases in women, with 240,000 new cases diagnosed annually and an annual mortality rate of 150,000 from ovarian cancer across the globe (1). In the US alone, $\sim 22,000$ ovarian cancer cases are diagnosed and $\sim 14,000$ individuals succumb to mortality annually (2). Ovarian cancer is often referred to as a 'silent killer' as $80 \%$ of patients show no symptoms of the disease until an advanced stage if the cancer develops within the ovaries (3). It has been reported in the last few decades that $20 \%$ of patients diagnosed with ovarian cancer at an early stage have $>90 \%$ survival rate (3). Although efficient surgical interventions and the use of different combinations of anticancer drugs have shown promising results, the overall cure rate remains at only $30 \%$ (4). Due to the distinct biology of ovarian cancer, the selection of treatment options and effective drug combinations remain limited (4). Therefore, there is an urgent requirement to examine novel and more effective drugs for the treatment of ovarian cancer. Consistently, natural products have shown a diverse range of human health-promoting properties since times immemorial (5). Natural products are mainly secondary metabolites synthesized by living organisms to adapt to and survive under extreme environmental conditions. Due to the tendency of these secondary metabolites to interact with enzymes, receptors and other cellular entities, they exhibit appropriate drug properties (5). Statistical data between 1981 and 2010 have revealed that a number of natural products have been established as sources of novel drugs (5). Furthermore, the diverse molecular scaffolds of natural products often exhibit drug properties (6), and are considered essential for combinatorial $(7,8)$ and diversity-oriented synthesis (9) for the development of effective bioactive molecules. 
It has been reported that, of the 155 small-molecule anticancer drugs, the number of drugs that are natural products and/or synthesized from natural products accounts for $72.9 \%$ (10). Among these, natural sources from plants represent an important source of anticancer drugs; and several plant-derived compounds are currently used as anticancer drugs or are being assessed in clinical trials (11-13). Isoflavones are plant secondary metabolites with substantial pharmacological potential (14). They are common food ingredients and, therefore, are considered safe $(14,15)$. Several studies have reported that the consumption of isoflavonoids is inversely proportional to the risk of cancer $(14,15)$. Daidzein (7,4-dihydroxyisoflavone), a flavone of plant origin, has been reported to exhibit anticancer activity against several types of cancer, including breast and ovarian cancer. However, the anticancer effect of daidzein has not been thoroughly investigated and the detailed mechanisms remain to be elucidated. In addition, the RAF/mitogen-activated protein kinase kinase (MEK)/extracellular signal-regulated kinase (ERK) signaling pathways have been reported to be crucial in the tumorigenesis and progression of several types of cancer, including ovarian cancer (16). Therefore, the present study was designed to examine the anticancer activity of daidzein in vitro and in vivo, and to attempt to investigate the underlying mechanisms.

\section{Materials and methods}

Chemicals, reagents and cell cultures. Daidzein (98\% pure by HPLC) and other chemicals were procured from Sigma-Aldrich; Merck Millipore (Darmstadt, Germany) unless otherwise mentioned. The normal human ovarian epithelial cell line (Moody), and the Caov-3, OVACAR-3, SKOV3 and A2780 human ovarian tumor cell lines were obtained from the Type Culture Collection of Chinese Academy of Sciences (Shanghai, China). For culturing of the cells, RPMI-1640 media was used, which also contained fetal bovine serum (Thermo Fisher Scientific, Inc., Waltham, MA, USA) (10\%) and appropriate antibiotics (streptomycin $100 \mu \mathrm{g} / \mathrm{ml}$ and penicillin $\mathrm{G} 100 \mathrm{U} / \mathrm{ml}$ ). The cultures were maintained in an atmosphere containing $5 \% \mathrm{CO}_{2}$ at $37^{\circ} \mathrm{C}$.

CCK-8 assay for the assessment of cell viability. The cell viability of the non-cancerous ovarian cells (Moody) and the ovarian cancer cells (SKOV3) was determined using a CCK-8 assay. In brief, $5 \times 10^{3}$ cells were seeded in a 96-wel lplate and maintained at $37^{\circ} \mathrm{C}$ in a humidified $5 \% \mathrm{CO}_{2}$ atmosphere. Following incubation overnight, the cells were treated with varied doses of daidzein $(0,0.78,1.56,3.12,6.25,12.5,25,25$, 50,100 and $200 \mu \mathrm{M}$ ) for $24 \mathrm{~h}$. Subsequently, $10 \mu \mathrm{l}$ of CCK-8 was added into each well and incubated again at $37^{\circ} \mathrm{C}$ for $1 \mathrm{~h}$. The optical density (OD) at $\mathrm{OD}_{450} \mathrm{~nm}$ was determined using a microplate spectrophotometer (Bio-Rad Laboratories, Inc., Hercules, CA, USA) and reported as a percentage of the control.

AO/EB, DAPI and Annexin V/propidium iodide (PI) staining. The SKOV 3 cells were cultured at a density of $2 \times 10^{5}$ cells/well in six-well plates, subjected to varied doses of daidzein $(0,10$, 20 and $40 \mu \mathrm{M})$ and incubated for $24 \mathrm{~h}$ at $37^{\circ} \mathrm{C}$. The cells were then stained with a mixture of $\mathrm{AO}$ and $\mathrm{EB}$. The stained cells were examined under the fluorescent microscope. DAPI staining was performed by incubating the cells in 6-well plates and treated with different concentrations of daidzein $(0,10$, 20 and $40 \mu \mathrm{M})$. The cells were then washed with PBS, fixed in formaldehyde (10\%) and the washed with PBS. The cells were subjected to DAPI staining and then examined using fluorescence microscopy. For the estimation of apoptosis, the cells were subjected to Annexin V/PI double staining, using a similar procedure to that used for DAPI staining, and was investigated using flow cytometry.

Determination of mitochondrial membrane potential (MMP). The SKOV 3 cells were cultured at $2 \times 10^{5}$ cells/well in a six-well plate and incubated for $24 \mathrm{~h}$ with $0,10,20$ and $40 \mu \mathrm{M}$ daidzein at $37^{\circ} \mathrm{C}$ in an atmosphere consisting of $5 \% \mathrm{CO}_{2}$ and $95 \%$ air. The cells from all samples were then harvested and subjected to washing with PBS, and mixed with $500 \mu \mathrm{l}$ of $\mathrm{DiOC}_{6}(1 \mu \mathrm{mol} / \mathrm{l})$ for the estimation of MMP at $37^{\circ} \mathrm{C}$ for $30 \mathrm{~min}$. Subsequently, flow cytometry was used to examine the cell samples.

Cell cycle analysis. Following staining with PI, the cells were subjected to flow cytometry for the evaluation of cell cycle phase distribution. Briefly, the SKOV3 cells were seeded at a density of $2 \times 10^{5}$ and treated with varied doses of daidzein $(0,10,20$ and $40 \mu \mathrm{M})$ for $24 \mathrm{~h}$, following which the cells were haryested and placed in ethanol $(70 \%)$ at $4^{\circ} \mathrm{C}$. Following overnight incubation, the cells were harvested and centrifuged for $10 \mathrm{~min}$ at $800 \mathrm{x} \mathrm{g}$, washed in PBS and finally suspended in PBS $(250 \mathrm{ml})$. This was followed by RNase treatment for $\sim 20 \mathrm{~min}$. The cell cycle phase was then determined using flow cytometry following PI staining.

Cell migration assay. The migration of SKOV3 cells was determined using a wound healing assay. Briefly, the cells were cultured until confluence. The SKOV3 cells were then treated with different concentrations of daidzein $(0,5,10$ and $20 \mu \mathrm{M})$ and a scratch was introduced to the cell culture using a sterile pipette tip, followed by incubation for $24 \mathrm{~h}$. The wound healing capacity of the daidzein-treated cells was determined by comparing the wound length with that of the untreated control cells.

Analysis of protein expression by western blot analysis. Total proteins from the untreated and daidzein-treated cells were isolated in RIPA lysis buffer. The concentrations of the proteins in each assay were determined by BCA assay. Equal quantities of protein extracts from each group were then run on SDS-PAGE gels (10\%) and then transferred onto a polyvinylidene fluoride membrane. This was followed by blocking with 5\% non-fat milk and incubation at room temperature for $1 \mathrm{~h}$. The membranes were then incubated with a specific primary antibody (MEK, sc-6250; pMEk, sc-136542; ERK, sc-93; pERK, sc-7383; MMP-9, sc-176046; MMP-2 sc-176407; pCdc25c, sc-327; pCd25c, sc-56296; pCdc2, sc-12340; Cdc2, sc-54; Cyclin B1, sc-70898; p27, sc-1641; p21, sc-122305; p53, sc-47698; Cyto C, sc-65396; Bax, 623621; BCl2, sc-509; pro-caspase-3, sc-113427; cleaved caspase-3, sc-176260; pro-caspase-9, sc-81650; cleaved caspase-9, sc-81663; PARP, sc-8007; Cleaved PARP, sc-23461; Actin, sc-58673; GAPDH, sc-47724) overnight at $4^{\circ} \mathrm{C}$ (all 1:1,000; 
Santa Cruz Biotechnology, Inc., Dallas, TX, USA), followed by incubation with horse radish peroxidase-conjugated anti-rabbit secondary antibody $(1: 1,000 ;$ sc-2372; Santa Cruz Biotechnology, Inc.) for $1 \mathrm{~h}$ at room temperature. The protein bands of interest were visualized using an ECL Advanced Western Blot Detection kit (GE Healthcare Life Sciences, Uppsala, Sweden).

In vivo xenograft experiment. For the xenograft study, the whole procedure was performed as per the animal ethics guidelines and was approved by the animal ethics committee of Huai'an First People's hospital, Nanjing University (Huai'an, China; approval no. NUX278/2016; 12 May 2016). The six-week old, immunodeficient nude mice (14 male and 14 female) weighing 25-30 g were procured from the Animal Centre of Nanjing University and housed in sterile stainless-steel cages in a 12-h light:dark cycle at $22^{\circ} \mathrm{C}$ and with $\sim 50 \%$ relative humidity. The mice received a subcutaneous injection in the left flank ofSKVO3 cells $\left(5 \times 10^{6}\right)$. As the tumors became visible, the mice were administrated i.p. with DMSO (0.1\%), dissolved daidzein and diluted with normal saline at concentrations of 10,20 and $40 \mu \mathrm{g} / \mathrm{kg}$ body weight. The doses were administrated three times each week (Monday, Thursday and Saturday) and the control mice received $0.1 \%$ DMSO in normal saline only. The tumor volume and size were estimated every week using standard procedures. During and at the end of the 4-week period, the mice were sacrificed by inhaling of deep anesthesia with isoflurane $(2.5 \%$ of the oxygen supplied for $2 \mathrm{~h}$ ) and organs were collected for further experimentation. The study was terminated at the 4-week end point as a marked difference was observed in the tumor volume and weight at this point.

Statistical analysis. The data are presented as the mean \pm standard deviation. Statistical significance and $\mathrm{IC}_{50}$ values were analyzed using GraphPad Prism Demo, Version 5 (GraphPad Software, Inc., San Diego, CA, USA). Student's t-test was used for comparison between two samples, and one-way analysis of variance followed by Tukey's test was used for comparisons between more than two samples for statistical analysis. $\mathrm{P}<0.01$ was considered to indicate a statistically significant difference.

\section{Results}

Daidzein exerts antiproliferative effects on SKOV3 cells. The antiproliferative effect of daidzein against a panel of human ovarian cancer cells and normal (Moody) ovarian cells was evaluated using a CCK-8 assay (Table I). The results indicated that, of all ovarian cancer cell lines, daidzein exerted the most marked dose-dependent antiproliferative effects on SKVO3 cells. However, daidzein was found to be less cytotoxic against the normal cells (Fig. 1A). The $\mathrm{IC}_{50}$ of daidzein against the SKOV3 cells was $20 \mu \mathrm{M}$, compared with the $\mathrm{IC}_{50}$ of $100 \mu \mathrm{M}$ for the normal ovarian cells. In addition, daidzein affected the morphology of the SKOV3 cells (Fig. 1B). As daidzein exhibited the lowest $\mathrm{IC}_{50}$ against $\mathrm{SKOV} 3$ cells, subsequent experiments were performed using only this cell line. As the concentration of daidzein was increased, the SKOV3 cancer cells became rounder, shrunken and detached from the substratum (Fig. 1B), which are important morphological changes associated with apoptosis.
Table I. IC $_{50}$ values of daidzein against different ovarian cancer cell lines. Experiments were performed in three biological replicates.

\begin{tabular}{lc} 
Cell line & $\mathrm{IC}_{50}(\mu \mathrm{M})$ \\
\hline Caov-3 & 25 \\
OVACAR-3 & 25 \\
SKOV3 & 20 \\
A2780 & 40 \\
Moody (normal cell line) & 100 \\
\hline
\end{tabular}

$\mathrm{IC}_{50}$, half-maximal inhibitory concentration.

Daidzein triggers mitochondrial apoptosis. As daidzein induced morphological changes in the SKOV3 cells characteristic of apoptosis, DAPI and AO/EB staining were performed. The results indicated that daidzein induced apoptosis of the SKOV3 cells, as evident from the increasing number of nuclei stained white in the case of DAPI staining (Fig. 2A) and showing orange fluorescence in the case of $\mathrm{AO} / \mathrm{EB}$ staining (Fig. 2B). To estimate the apoptotic cell populations, Annexin V/PI double staining was performed, and the results indicated that the proportion of apoptotic cells increased with the increase in the concentration of daidzein (Fig. 2C). The apoptotic cell populations were $5.2,11.3,35.7$ and $48.8 \%$ at the concentrations of $0,10,20$ and $40 \mu \mathrm{M}$ daidzein, respectively. Subsequently, the effect of different concentrations of daidzein on MMP was determined. The results indicated that daidzein caused a reduction in the MMP of SKOV3 cells in a dose-dependent manner (Fig. 3A). Mitochondrial injury causes the release of cytochrome $c$ (cyt $c$ ) into the cytoplasm and promotes apoptotic factors, which activate the caspase cascade and mitochondria-triggered apoptosis (14). To investigate whether daidzein induces apoptosis via this mechanism in the SKOV3 cells, the expression levels of cyt $c$, B-cell lymphoma 2-associated X protein (Bax), $\mathrm{BH} 3$ interacting-domain death agonist (Bid), caspase-3, -9 and -8, and poly (ADP-ribose) polymerase (PARP) were determined using western blot analysis (Fig. 3B). The results showed that daidzein increased the cytosolic levels of cyt $c$, Bax, cleaved caspase- 3 and -9 , and cleaved PARP, compared with levels in the control (Fig. 3C).

Daidzein induces $62 / M$ cell cycle arrest. Cell cycle arrest is one of the important mechanisms by which anticancer agents exert their inhibitory effects. Therefore, the present study also determined the effect of daidzein treatment on cell cycle phase dissemination of the SKOV3 cells (Fig. 4A). The results indicated that the number of SKOV3 cells was significantly enhanced in the $\mathrm{G} 2$ phase at doses of $0-40 \mu \mathrm{M}$ daidzein, leading to G2/M cell cycle phase arrest (Fig. 4A). Additionally, the populations of SKOV3 cells in the G2 phase were marginally enhanced at the dosage of $10 \mu \mathrm{M}$, moderately enhanced at $20 \mu \mathrm{M}$ and markedly increased at $40 \mu \mathrm{M}$, indicating the dose-dependent effect of daidzein. Western blot analysis was then performed to investigate the effect of daidzein on the expression of $\mathrm{G} 2 / \mathrm{M}$ cell cycle controlling proteins, including 

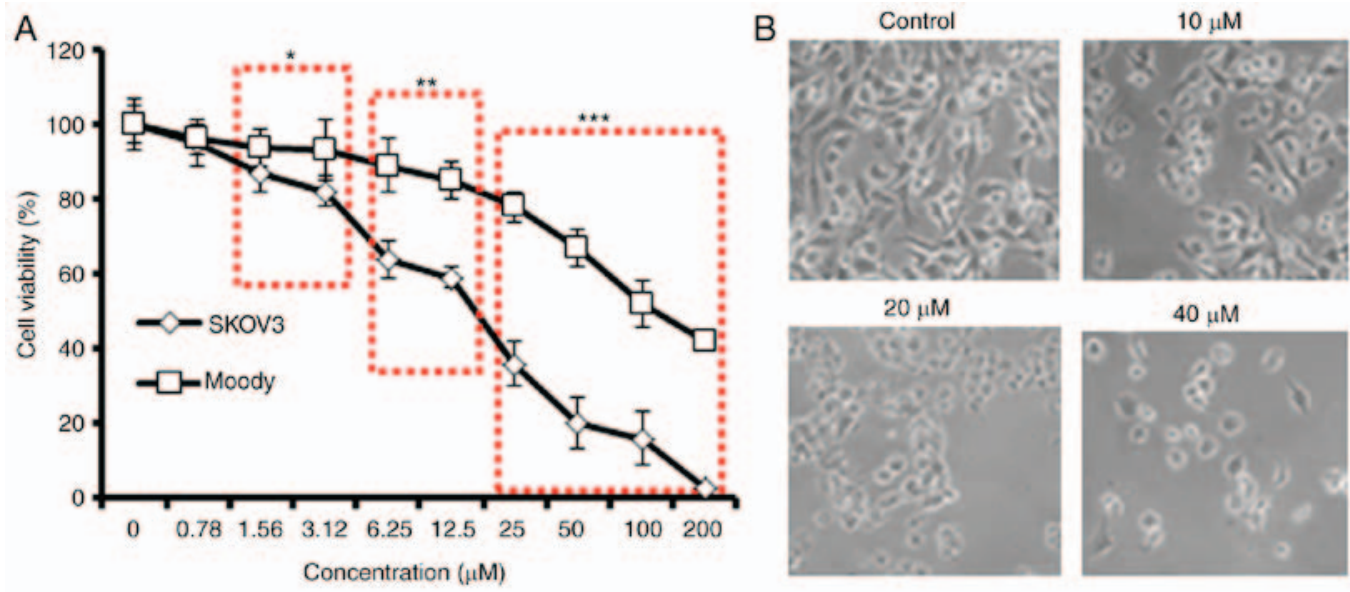

Figure 1. Effects of daidzein on cell viability and morphology. Effects of varied concentrations of daidzein on (A) cell viability and the (B) morphology of SKOV3 ovarian cancer and normal (Moody) ovarian cells (magnification, x200). The experiments were performed in triplicates and results are presented as the mean \pm standard deviation $\left({ }^{*} \mathrm{P}<0.01,{ }^{* * *} \mathrm{P}<0.001\right.$ and $\left.{ }^{* * * *} \mathrm{P}<0.0001\right)$.
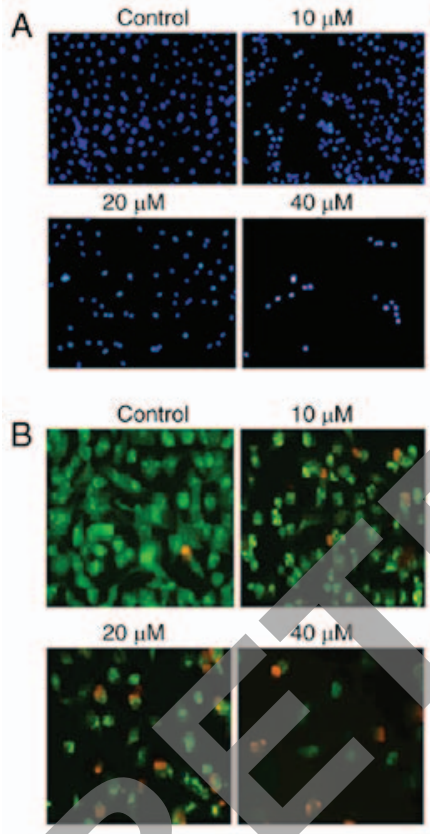

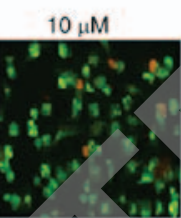
$40 \mu \mathrm{M}$

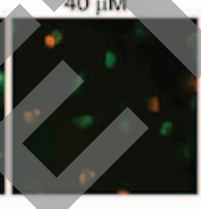

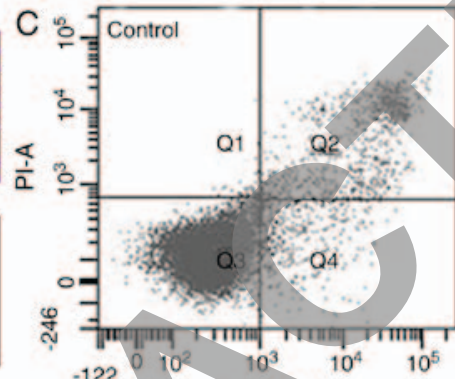
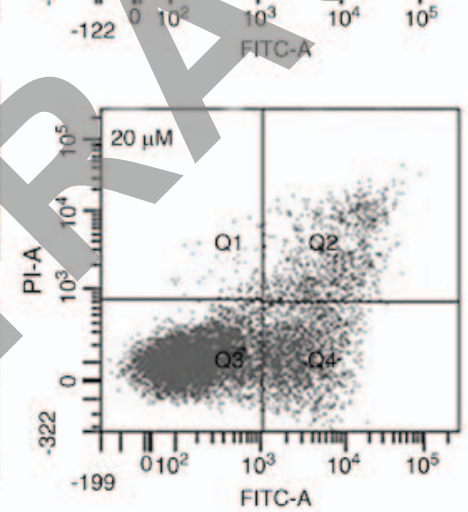
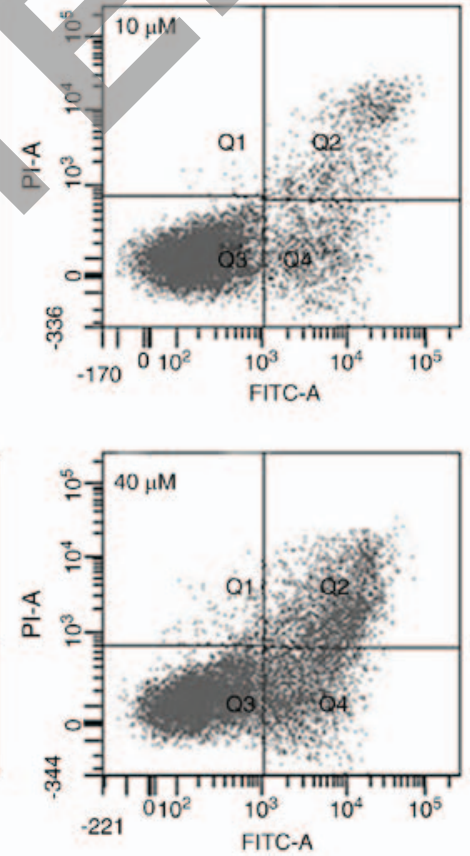

Figure 2. Effects of daidzein on apoptosis. Effects of the indicated doses of daidzein on (A) induction of apoptosis using (A) DAPI staining and (B) AO/EB staining (magnification, x200). (C) Determination of apoptotic cell populations using Annexin V/PI staining in SKOV3 cells. Results are presented as the mean of triplicate experiments. PI, propidium iodide.
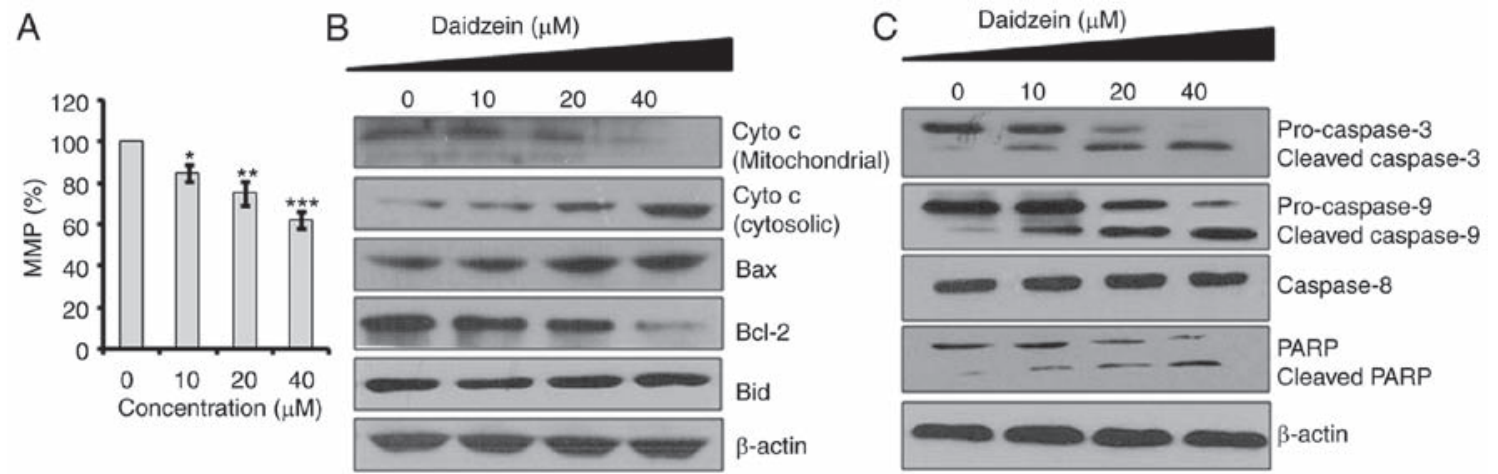

Figure 3. Effects of daidzein on MMP. Effects of the indicated doses of daidzein on (A) MMP, and the expression of (B) cyto $c$, Bax, Bcl-2 and Bid, (C) caspase-3, caspase-9, caspase-8 and PARP in SKOV3 cells. The experiments were performed in triplicate and presented as the mean \pm standard deviation ( $\mathrm{P}<0.01,{ }^{* *} \mathrm{P}<0.001$ and ${ }^{* * *} \mathrm{P}<0.0001$, vs. control). MMP, mitochondrial membrane potential; cyto $c$, cytochrome $c$; Bcl-2, B-cell lymphoma 2; Bax, Bcl-2-associated X protein; Bid, BH3 interacting-domaindeath agonist; PARP, poly (ADP-ribose) polymerase. 


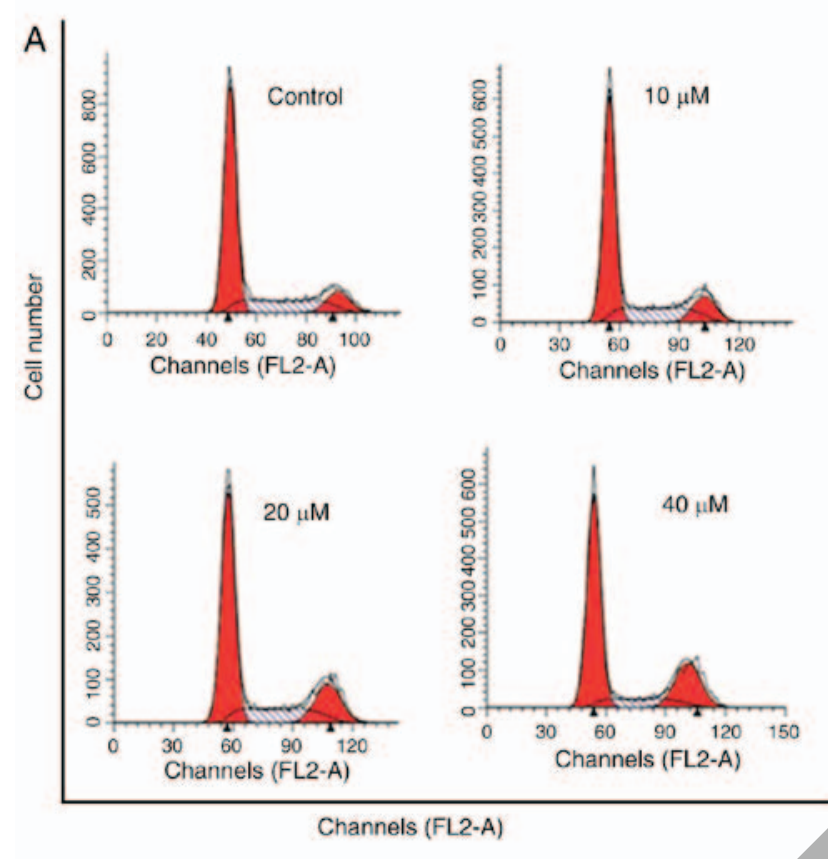

B
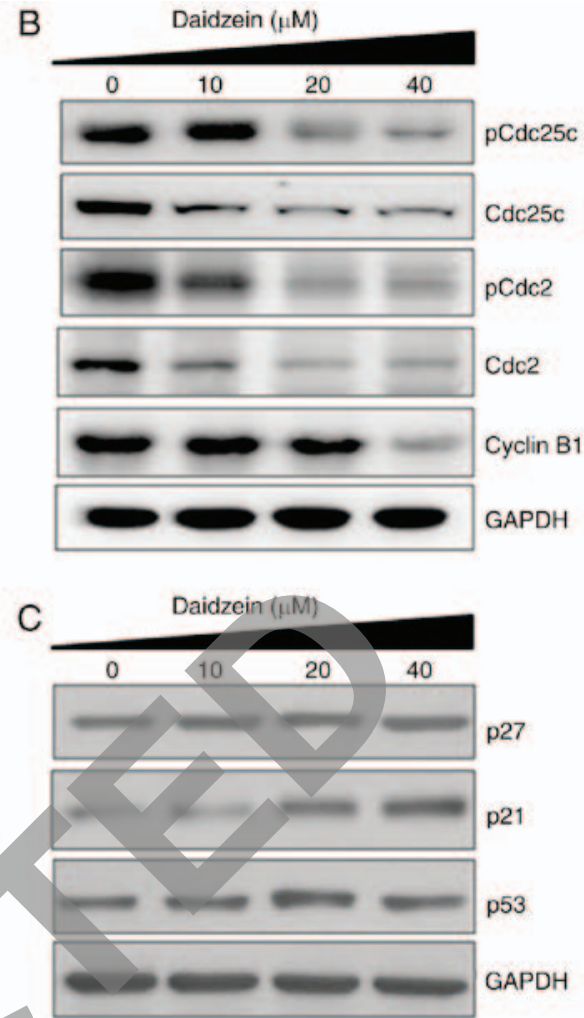

Figure 4. Effect of daidzein on cell cycle distribution. (A) Effects of indicated doses of daidzein on cycle distribution were determined using flow cytometry. Expression levels of (B) pCdc25c, Cdc25c, pCdc2, Cdc2 and cyclin B1, and (C) p21, p27 and p53. The experiments were performed in triplicate. pCdc25c, phosphorylated $\mathrm{Cdc} 25 \mathrm{c}$; pCdc2, phosphorylated Cdc2.

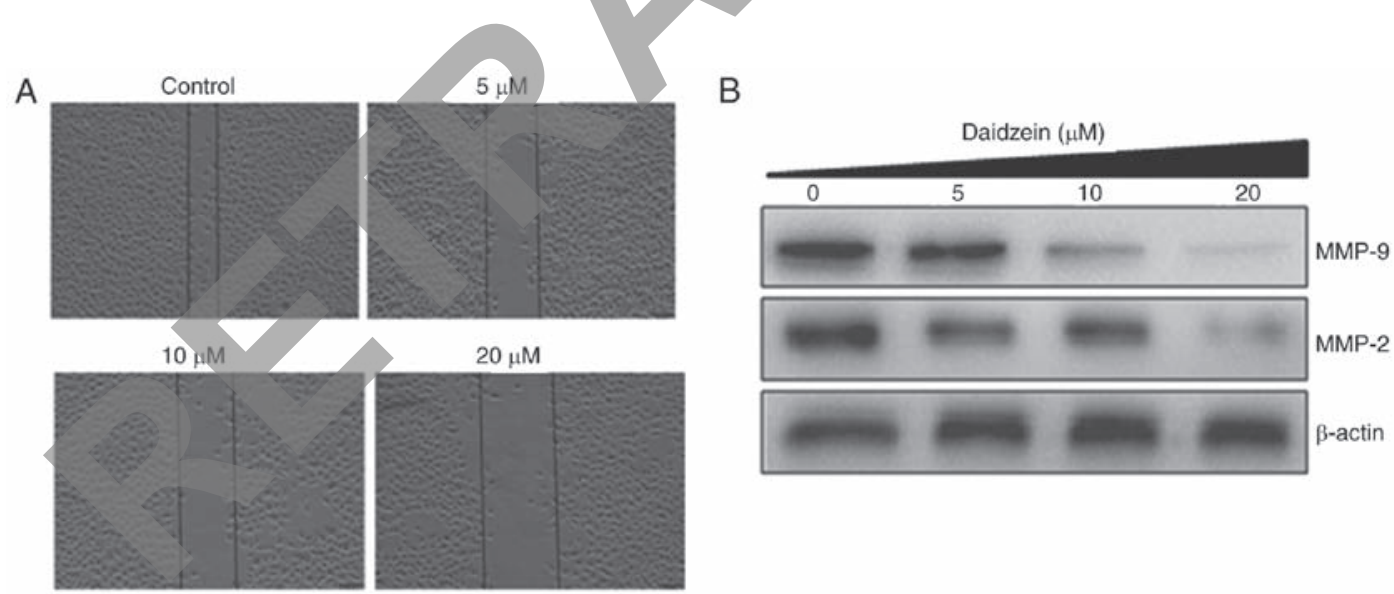

Figure 5. Effect of daidzein on migration of SKOV3 cells. (A) Migration of SKOV3 cells treated with varied doses of daidzein as indicated. Daidzein inhibited SKOV3 cell migration, as determined using a Transwell assay performed for $24 \mathrm{~h}$. (B) Expression levels of MMP-2 and MMP-9 in daidzein-treated SKOV3 cells were determined using western blot analysis. Experiments were repeated three times. MMP, matrix metalloproteinase.

cyclin B1, Cdc25c and Cdc2, in SKOV3 cells. Treatment of the SKOV3 cells with daidzein led to a concentration-dependent reduction in the protein levels of pCdc25c (Ser216), Cdc25c, pCdc2 (Tyr15), Cdc2 and cyclin B1 in the SKOV3 cells (Fig. 4B). Daidzein also caused an increase in the expression of $\mathrm{p} 21$, whereas no significant effect was reported on the expression levels of p27 or p53 (Fig. 4C).

Daidzein inhibits cell migration. The present study also examined whether daidzein can inhibit the migration of SKOV3 cancer cells at the different concentrations using a wound-healing assay. The results of the wound-healing assay showed that daidzein reduced the migratory capability of the SKOV3 cells in a dose-dependent manner. In the control group, the cells exhibited the capacity to migrate, whereas treatment led to cells showing reduced potential to migrate, as shown in Fig. 5A. Additionally, daidzein caused a reduction in the expression levels of MMP-9 and MMP-2 in a dose-dependent manner (Fig. 5B).

Daidzein inhibits the RAF/MEK/ERK signaling pathway. The RAF/MEK/ERK signaling pathway has been shown to be important in the tumorigenesis and progression of several types of cancer, including ovarian cancer. Therefore, the 


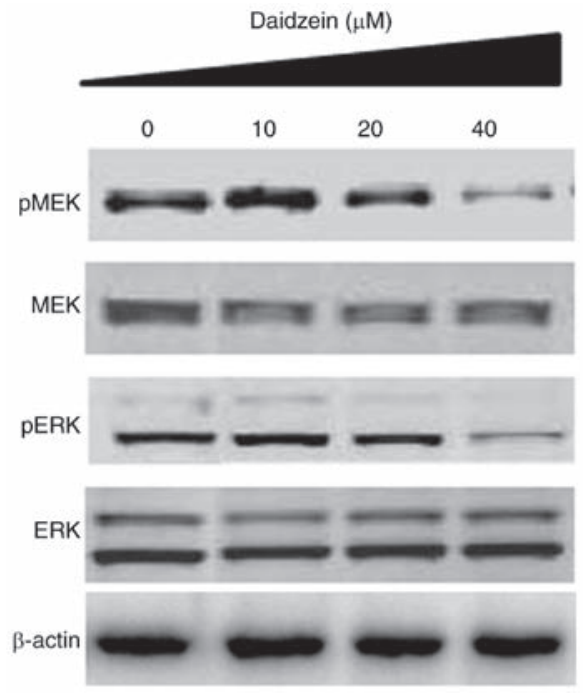

Figure 6. Effect of daidzein on the RAF/MEK/ERK signaling pathway. Effects of the indicated doses of daidzein on the expression of RAF/MEK/ERK signaling pathway proteins are shown. Experiments were repeated three times. MEK, mitogen-activated protein kinase kinase; ERK, extracellular signal-regulated kinase; pMEK, phosphorylated MEK; pERK, phosphorylated ERK.

present study evaluated the effect of various concentrations of daidzein on the RAF/MEK/ERK cascade. The results indicated that daidzein suppressed the phosphorylation of MEK and ERK in a dose-dependent manner (Fig. 6).
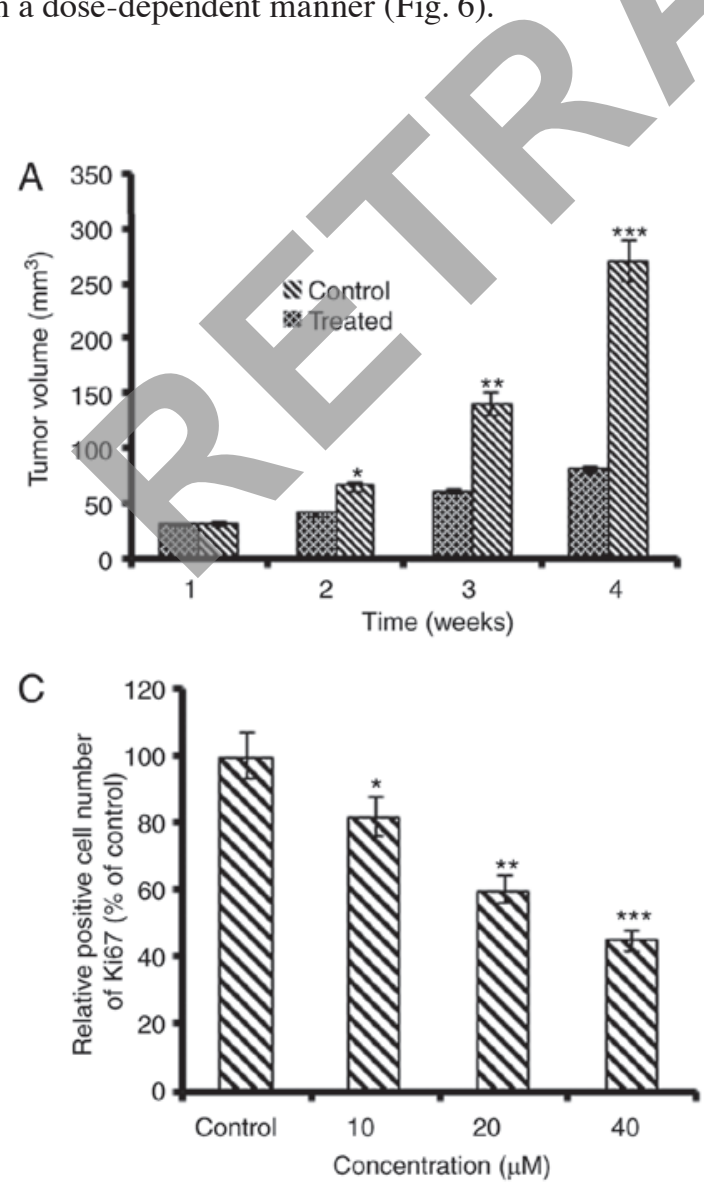

Daidzein inhibits tumor growth in vivo. To examine the anticancer effects of daidzein in vivo, it was assessed in nude mice xenograft models. The results revealed that SKOV3 tumor growth was significantly suppressed by daidzein administration, compared with that in the control group. At the end of the 4-week period of daidzein treatment, the average tumor growth and volume in the untreated control group were considerably higher than those in the treated groups (Fig. 7A and B). In addition, the in vivo growth inhibitory potential was concentration- and time-dependent. The protein expression level of $\mathrm{Ki}-67$ in the xenografted tumors was downregulated and that of cleaved caspase-3 was enhanced following daidzein treatment (Fig. 7C and D).

\section{Discussion}

Of all forms of gynecological cancer, ovarian cancer is responsible for the highest mortality rate, mainly due to diagnosis being at advanced stages (17). Although the majority of patients react to debulking surgery and combinatorial therapy using taxane and platinum, there is frequent relapse of the disease due to intrinsic and acquired resistance. Therefore, novel options are required for the improved management and treatment of this disease at diagnosis and/or to offer an effective second line treatment. In the present study, the anticancer effects of daidzein against ovarian cancer cells were investigated. The results indicated that daidzein significantly suppressed the growth $\left(\mathrm{IC}_{50} 20 \mu \mathrm{M}\right)$ of SKOV3 cells. However, daidzein showed comparatively less cytotoxicity $\left(\mathrm{IC}_{50} 100 \mu \mathrm{M}\right)$
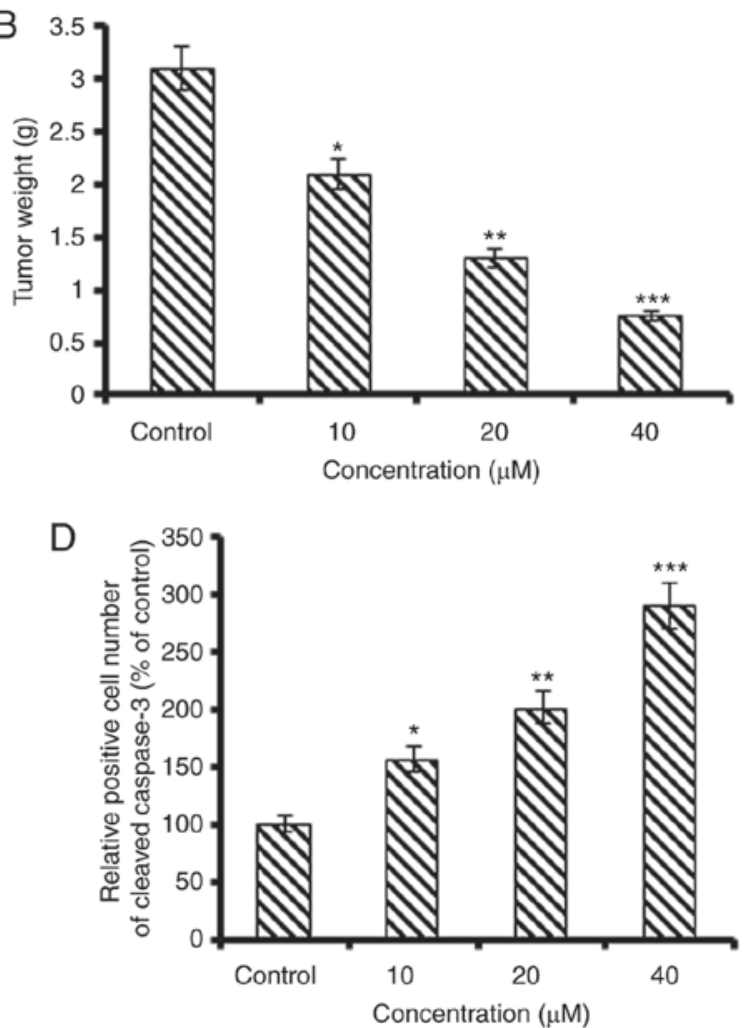

Figure 7. Daidzein inhibits SKOV3 tumor growth in vivo. (A) Tumor volume and (B) tumor weight were measured at indicated time intervals and doses of daidzein. (C) Relative percentages of Ki67-positive cells and (D) cleaved caspase-3. The experiments were performed in triplicate and data are presented as the mean \pm standard deviation $\left({ }^{*} \mathrm{P}<0.01,{ }^{* *} \mathrm{P}<0.001\right.$ and ${ }^{* * * *} \mathrm{P}<0.0001$, vs. control). 
towards the normal ovarian cells, indicating that daidzein selectively targeted cancer cells (Fig. 1). These results are in accordance with those of previous studies, in which daidzein has been shown to suppress the proliferation of several types of cancer $(14,18-20)$. Daidzein treatment also triggered several morphological changes in SKOV3 cells, which are characteristic of apoptosis. Following this observation, DAPI and $\mathrm{AO} / \mathrm{EB}$ staining were performed to examine whether daidzein induces the apoptosis of SKOV3 cells. It was observed that daidzein increased the DNA fragmentation, observed using DAPI, and led to an increase in orange fluorescence following $\mathrm{AO} / \mathrm{EB}$ staining, which are changes indicative of apoptosis. These results were substantiated by the results of Annexin V/IP staining, which showed that daidzein treatment led to a significant increase in the apoptotic cell populations of SKOV3 cells (Fig. 2). These results are supported by a previous report that daidzein induces apoptosis in cancer cells (20). To determine whether the apoptosis follows the mitochondrial route, the present study determined the effect of daidzein on MMP, and it was observed that daidzein significantly decreased MMP, which was associated with upregulation in the expression levels of cyt $c$, Bax, cleaved caspase-3 and -9 , and cleaved PARP, compared with levels in the control SKOV3 cells (Fig. 3). It has been reported that drugs with apoptosis-inducing properties exhibit the potential to minimize potential drug resistance (21) and the results of the present study clearly indicated that daidzein had apoptosis-inducting properties, suggesting that daidzein may be an important lead molecule for the treatment of ovarian cancer. In addition to apoptosis, cell cycle arrest is another mechanism by which anticancer agents inhibit cancer cell proliferation. The results of the present study revealed that daidzein caused G2/M cell cycle arrest. Apoptotic cell death is triggered when explicit checkpoints are arrested during cell cycle. Consistent with this, several anticancer agents cause cell cycle arrest and have been found to be clinically effective for cancer treatment (22). The increase of cells in the G2/M phase prevents cells undergoing mitosis $(23,24)$. Treatment of the SKOV3 cells with daidzein led to incomplete cell division, revealing that cells at the $\mathrm{G} 2 / \mathrm{M}$ phase were unsuccessful in entering and undergoing mitosis due to the suppression of $\mathrm{G} 2 / \mathrm{M}$ regulatory proteins, including cyclin $\mathrm{B} 1, \mathrm{Cdc} 25 \mathrm{C}$ and Cdc2 (Fig. 4). p21WAF1 is an important inhibitor in modulating cell cycle progression (25). There is evidence indicating that p21WAF1 is linked to suppression of the expression of the $\mathrm{Cdc} 2 /$ cyclin B1 complex $(26,27)$. The results of the present study revealed that daidzein suppressed the expression of cyclin $\mathrm{B} 1, \mathrm{Cdc} 25 \mathrm{C}$ and $\mathrm{Cdc} 2$, and increased the protein expression levels of p21/WAF1 (Fig. 5B). However, daidzein had no significant effect on the expression of either p27 or p53. These results indicated that daidzein stimulated the expression of p21WAF1, which in turn triggered $\mathrm{G} 2 / \mathrm{M}$ phase arrest of the p53-independent pathway. Cell migration is one of the important characteristics of cancer cell progression and metastasis (28), and the inhibition of cell migration may be beneficial in suppressing metastasis under in vivo conditions. In the present study, it was observed that daidzein inhibited the migration of SKOV3 cells, which was associated with a concomitant decrease in the expression of MMP-2/9 (Fig. 5). Dysregulation of the RAS/ERK pathway is common in all histotypes of ovarian cancer, and targeting this pathway may offer a novel alternative $(29,30)$. Therefore, the present study determined the effect of daidzein on the RAF/MEK/ERK pathway, which revealed that daidzein inhibited the phosphorylation of MEK and ERK, indicating daidzein may be a potential candidate for targeting this pathway (Fig. 6). However, daidzein-induced G2/M cell cycle arrest may not be linked to inhibition of the RAF/MEK/ERK pathway. Due to the complexity and cross talk between the pathways, daidzein may induce G2/M2 cell cycle arrest by a mechanism other than inhibition of RAF/MEK/ERK pathway and further investigations are require to elucidate this.

The in vivo evaluation of daidzein revealed that it markedly inhibitedSKOV3 ovarian tumor growth, compared with that in the untreated control group and induced with no apparent toxicity. The Ki67 and cleaved caspase-3 proteins are considered important cellular markers of proliferation and apoptosis, respectively (31). The decrease in Ki67-positive cells and significant increase in the expression of cleaved caspase- 3 suggested that daidzein effectively inhibited ovarian cancer cell growth in vivo and maybe an important lead molecule.

Taken together, the results of the present study indicated that daidzein exerted significant anticancer effects towards SKOV3 cancer cells by inducing mitochondrial apoptosis and cell cycle arrest. Daidzein also inhibited tumor growth in vivo, indicating that it may offer potential as a lead molecule in the management of ovarian cancer and warrants further investigation.

\section{Competing interests}

The authors declare that they have no competing interests.

\section{References}

1. Siegel R, Ma J, Zou Z and Jemal A: Cancer statistics, 2014. CA Can J Clin 64: 9-29, 2014.

2. Bast RC Jr, Urban N, Shridhar V, Smith D, Zhang Z, Skates S, Lu K, Liu J, Fishman D and Mills G: Early detection of ovarian cancer: Promise and reality. Cancer Treat Res 107: 61-97, 2002.

3. Berek JS: Ovarian cancer. In: Practical Gynecologic Oncology. Berek JS, Hacker NF (eds). Lippincott Williams \& Wilkins, Philadelphia, PA, pp443e511, 2005.

4. Bast RC Jr, Hennessy B and Mills GB: The biology of ovarian cancer: New opportunities for translation. Nat Rev Cancer 9: 415-428, 2009.

5. Newman DJ and Cragg GM: Natural products as sources of new drugs over the 30 years from 1981 to 2010. J Nat Prod 75: 311-35, 2012.

6. Over B, Wetzel S, Grütter C, Nakai Y, Renner S, Rauh D and Waldmann $\mathrm{H}$ : Natural-product-derived fragments for fragment-based ligand discovery. Nat Chem 5: 21-28, 2013.

7. Clough J, Chen S, Gordon EM, Hackbarth C, Lam S, Trias J, White RJ,Candiani G, Donadio S, Romanò G, et al: Combinatorial modification of natural products: Synthesis and in vitro analysis of derivatives of thiazole peptide antibiotic GE2270 A: A-ring modifications. Biorg Med Chem Lett 13: 3409-3414, 2003.

8. Cordier C, Morton D, Murrison S, Nelson A and O'Leary-Steele C: Natural products as an inspiration in the diversity-oriented synthesis of bioactive compound libraries. Nat Prod Rep 25: 719-737, 2008 .

9. Newman DJ and Cragg GM: Natural products as sources of new drugs over the last 25 years. J Nat Prod 70: 461-477, 2007.

10. Cragg GM, Grothaus PG and Newman DJ: Impact of natural products on developing new anti-cancer agents. Chem Rev 109: 3012-3043, 2009.

11. Shah U, Shah R, Acharya S and Acharya N: Novel anticancer agents from plant sources. Chin J Nat Med 11: 16-23, 2013.

12. Zhang X, Chen LX, Ouyang L, Cheng Y and Liu B: Plant natural compounds: Targeting pathways of autophagy as anti-cancer therapeutic agents. Cell Prolif 45: 466-476, 2012. 
13. Liggins J, Bluck LJ, Runswick S, Atkinson C, Coward WA and Bingham SA: Daidzein and genistein contents of vegetables. British J Nutr 84: 717-725, 2000.

14. Liggins J, Bluck LJ, Runswick S, Atkinson C, Coward WA and Bingham SA: Bingham Daidzein and genistein content of fruits and nuts. J Nutr Biochem 11: 326-331, 2000.

15. Cancer Genome Atlas Research Network: Integrated genomic analyses of ovarian carcinoma. Nature 474: 609-615, 2011.

16. Siegel R, Naishadham D and Jemal A: Cancer statistics, 2012. CA Cancer J Clin 62: 10-29. 2012.

17. Ullah MF, Ahmad A, Bhat SH, Khan HY, Zubair H, Sarkar FH and Hadi SM: Simulating hypoxia-induced acidic environment in cancer cells facilitates mobilization and redox-cycling of genomic copper by daidzein leading to pro-oxidant cell death: Implications for the sensitization of resistant hypoxic cancer cells to therapeutic challenges. Biometals 29: 299-310, 2016.

18. Choi EJ and Kim GH: Daidzein causes cell cycle arrest at the G1 and G2/M phases in human breast cancer MCF-7 and MDA-MB-453 cells. Phytomedicine 15: 683-690, 2008.

19. Jin S, Zhang QY, Kang XM, Wang JX and Zhao WH: Daidzein induces MCF-7 breast cancer cell apoptosis via the mitochondrial pathway. Ann Oncol 21: 263-268, 2009.

20. Zhang M, Liu Y, Gao Y and Li S: Silibinin-induced glioma cell apoptosis by PI3K-mediated but Akt-independent downregulation of FoxM1 expression. Euro J Pharmacol 765: 346-354, 2015.

21. Lee HG, Park WJ, Shin SJ, Kwon SH, Cha SD, Seo YH, Jeong JH, Lee JY and Cho CH: Hsp90 inhibitor SY-016 induces G2/M arrest and apoptosis in paclitaxel-resistant human ovarian cancer cells. Oncol Lett 13: 2817-2822, 2017.

22. Gautier J, Solomon MJ, Booher RN, Bazan JF and Kirschner MW: cdc25 is a specific tyrosine phosphatase that directly activates p34 ${ }^{\text {cdc2 }}$. Cell 67: 197-211, 1991.
23. Sancar A, Lindsey-Boltz LA, Unsal-Kaçmaz K and Linn S: Molecular mechanisms of mammalian DNA repair and the DNA damage checkpoints. Ann Rev Biochem 73: 39-85, 2004.

24. Harper JW, Adami GR, Wei N, Keyomarsi K and Elledge SJ: The p21 Cdk-interacting protein Cip1 is a potent inhibitor of G1 cyclin-dependent kinases. Cell 75: 805-816, 1993.

25. Baus F, Gire V, Fisher D, Piette J and Dulić V: Permanent cell cycle exit in $\mathrm{G}_{2}$ phase after DNA damage in normal human fibroblasts. EMBO J 22: 3992-4002, 2003.

26. Hsu YL, Kuo PL, Lin LT and Lin CC: Asiatic acid, a triterpene, induces apoptosis and cell cycle arrest through activation of extracellular signal-regulated kinase and p38 mitogen-activated protein kinase pathways in human breast cancer cells. J Pharmacol Exp Ther 313: 333-344, 2005.

27. Sun SY, Hail N Jr and Lotan R: Apoptosis as a novel target for cancer chemoprevention. J Natl Can Inst 96: 662-672, 2004.

28. Singer G, Oldt R III, Cohen Y, Wang BG, Sidransky D, Kurman RJ and Shih IeM: Mutations in BRAF and KRAS characterize the development of low-grade ovarian serous carcinoma. J Natl Cancer Inst 95: 484-486, 2003.

29. Auner V, Kriegshäuser G, Tong D, Horvat R, Reinthaller A, Mustea A and Zeillinger R: KRAS mutation analysis in ovarian samples using a high sensitivity biochip assay. BMC Can 9: 111, 2009.

30. Scholzen T and Gerdes J: The Ki-67 protein: From the known and the unknown. J Cell Physiol 182: 311-322, 2000.

31. Noble P, Vyas M, Al-Attar A, Durrant S, Scholefield J and Durrant L: High levels of cleaved caspase-3 in colorectal tumour stroma predict good survival. Br J Cancer 108: 2097-2105, 2013.

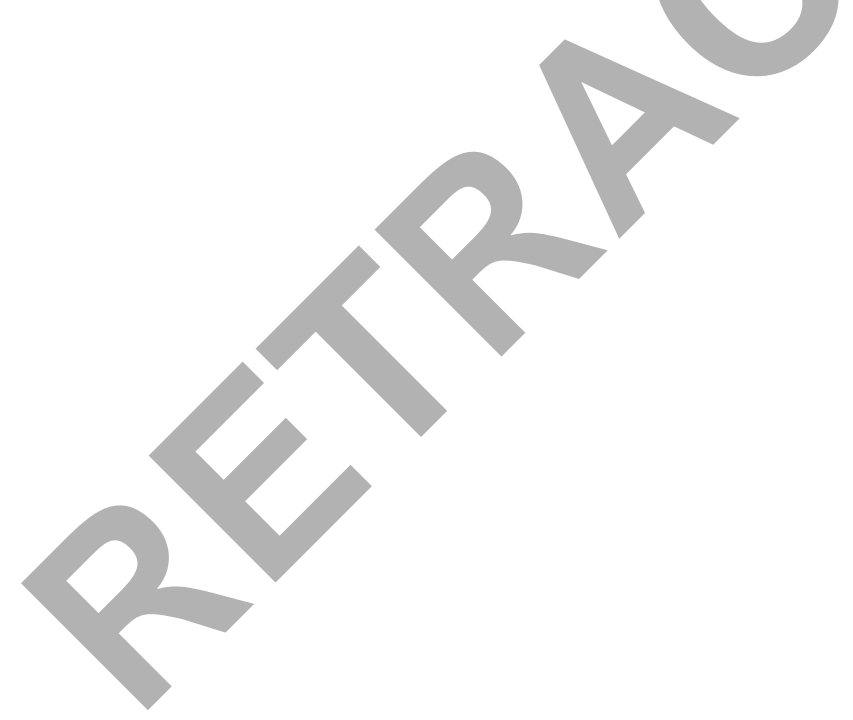

